\title{
Foamy Macrophage Deposition in Lymph Nodes Mimicking Lung Cancer Recurrence Diagnosed via Endobronchial Ultrasound-Guided Transbronchial Needle Aspiration
}

\author{
Qiaoling Zhou $^{\mathrm{a}} \quad$ Douglas G. West $^{\mathrm{a}} \quad$ Golda Shelley-Fraser $^{\mathrm{b}} \quad$ Andrew R.L. Medford $^{\mathrm{c}}$
}

Departments of a Thoracic Surgery and ${ }^{b}$ Pathology, Bristol Royal Infirmary, and c North Bristol Lung Centre, Southmead Hospital, Bristol, UK

\section{Established Facts}

- Mediastinal node sampling is more challenging with mediastinoscopy after previous surgical exploration and is more easily achieved with endobronchial ultrasound-guided transbronchial needle aspiration in this context.

- Oxidized cellulose haemostatic agents (OCHAs) are commonly used to control bleeding after surgical exploration with good effect, without the need for open exploration.

\section{Novel Insights}

- The use of OCHAs at a previous mediastinoscopy or surgical exploration may be associated with positron-emission tomography (PET)-avid lymph nodes and foamy macrophage deposition.

- It is therefore worth considering an OCHA inflammatory reaction in the differential diagnosis of PETavid mediastinal nodes, but resampling of the lymph nodes is important to avoid unnecessary oncological treatment, rather than assuming recurrent disease.

\section{Key Words}

Foamy macrophages - Oxidised cellulose haemostatic agents · Mediastinoscopy · Mediastinal lymphadenopathy · Endobronchial ultrasound-guided transbronchial needle aspiration

\section{Abstract \\ The radiological finding of mediastinal lymph node enlarge- ment following surgery for lung cancer often signifies lo- coregional recurrence. The use of oxidised cellulose haemo-}

static agents (OCHAs) during staging mediastinoscopy is common. We report a case of 18-fluorodeoxyglucose-avid subcarinal lymphadenopathy in a patient in whom OCHAs had been used at mediastinoscopy 5 months earlier. Histopathological examination of suspected nodal recurrence is facilitated by endobronchial ultrasound-guided transbronchial needle aspiration (EBUS-TBNA). The technique is particularly useful after previous mediastinoscopy, when repeat surgical exploration can be challenging. EBUS-TBNA samples showed extensive foamy macrophage deposition, with no evidence of malignancy. The association between the use

\section{KARGER 125}

(C) 2015 S. Karger AG, Basel

$0025-7931 / 15 / 0905-0426 \$ 39.50 / 0$

E-Mail karger@karger.com

www.karger.com/res
Dr. Andrew R.L. Medford

North Bristol Lung Centre

Level 6 Gate 10, Brunel Building, Southmead Hospital

Westbury-on-Trym, Bristol BS10 5NB (UK)

E-Mail andrewmedford@ hotmail.com 
of OCHAs and subsequent intranodal foamy macrophage deposition is new. Clinicians should consider this possibility in the differential diagnosis of mediastinal lymphadenopathy after surgical exploration, where OCHAs have been left in situ; it remains important to resample the lymph nodes before assuming disease recurrence to prevent unnecessary treatment.

(c) 2015 S. Karger AG, Basel

\section{Case Report}

A 66-year-old woman with a squamous cell carcinoma of the left upper lobe underwent a staging mediastinoscopy, which was complicated by bronchial artery bleeding at the left tracheobronchial angle (IASLC lymph node station left 4 [1]). This was controlled by packing the L4 and L7 node stations with oxidised cellulose gauze (Gelita-Cel ${ }^{\circledR}$, Gelita Medical GmbH, Eberbach, Germany), left in situ at the end of the procedure. A single $5 \times 1.25$ $\mathrm{cm}$ piece of Gelita-Cel was divided into three $1.7 \times 1.3 \mathrm{~cm}$ pieces for use. Haemostasis was achieved without open exploration. A premediastinoscopy positron-emission tomography/computed tomography (PET-CT) scan had shown no 18-fluorodeoxyglucose (18-FDG) uptake (apart from modest equivocal uptake in station 5) in the mediastinum (fig. 1). The mediastinoscopy biopsy samples showed no pathological abnormality (it was not practice at the time to sample station L5 as per the ESTS 2007 guidelines, and extended mediastinoscopic techniques were not available).

A pT2A pN0 squamous cell carcinoma was later excised with clear margins by open upper lobectomy. Pathological examination of lymph node stations R4, R7, L4, L6 and L9 (obtained at mediastinoscopy and intraoperative systematic mediastinal lymph node dissection) showed reactive changes only. She completed 2 cycles of carboplatin and vinorelbine chemotherapy postoperatively before complaining of breathlessness. A CT pulmonary angiogram showed no pulmonary emboli or other intrapulmonary new pathology, but did identify a $15-\mathrm{mm}$ subcarinal mass. A repeat PETCT scan confirmed a 15-mm 18-FDG-avid subcarinal station-7 node (standardised uptake value 7.7), with no uptake at distant sites (fig. 2).

As the patient had previously undergone a mediastinoscopy and surgical exploration, endobronchial ultrasound-guided transbronchial needle aspiration (EBUS-TBNA) was used to sample the subcarinal mass, with a convex probe ultrasound bronchoscope (Olympus BF-UC260FW, Olympus, Tokyo, Japan) and a 21-gauge needle under conscious sedation as previously described $[2,3]$. Microscopically, the biopsies comprised haemorrhagic tissue and sheets of foamy macrophages (fig. 3). Immunostaining for the macrophage marker CD68 was strongly positive. No evidence of malignancy or active infection was seen.

This florid macrophage response was considered most probably secondary to the use of OCHAs during previous mediastinoscopy. The patient remains well with no clinical or radiological evidence of recurrence over 12 months after EBUS, adding support to the above hypothesis.

Foamy Lymph Node Macrophage

Deposition after Haemostat

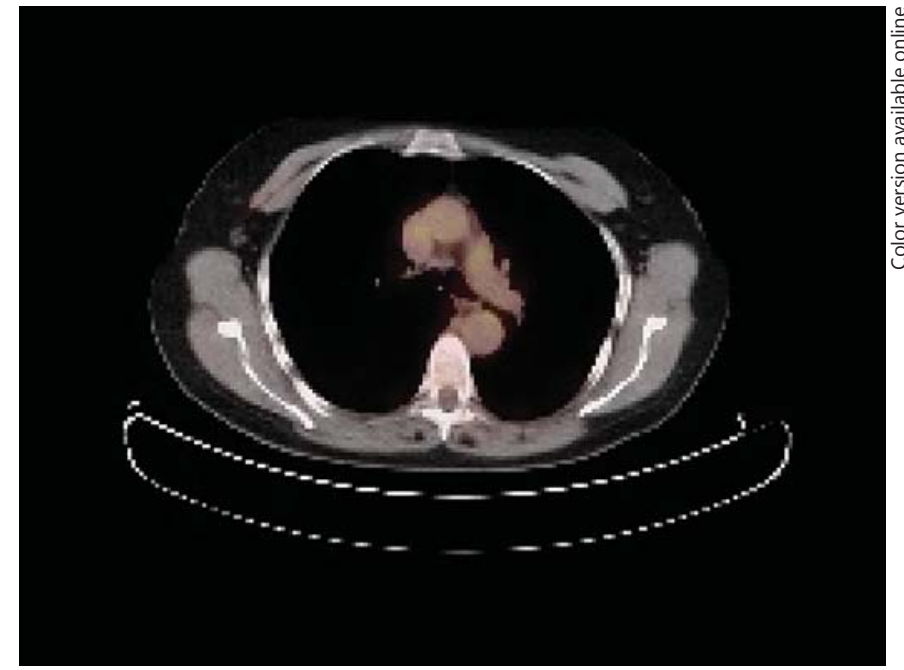

Fig. 1. Preoperative transverse PET sections of the subcarinal space.

\section{Discussion}

We describe foamy macrophage mediastinal lymph node deposition detected using EBUS-TBNA attributed to OCHA use at a previous mediastinoscopy, mimicking lung cancer recurrence. The 18 -FDG avidity of the subcarinal mass on PET-CT was an unusual characteristic of our case, falsely raising the suspicion of recurrent malignancy. Subcarinal lymphadenopathy was not seen on preoperative imaging (fig. 1). The subcarinal node mass was first identified at 4 months postoperatively. Therefore, there was a temporal correlation of the foamy macrophage accumulation in the subcarinal area only after the use of OCHAs.

OCHAs used in surgery induce haemostasis by providing a strong matrix for platelet adhesion and aggregation, accelerating the formation of clots [4]. They are designed to be left in situ after surgery. Reported events after the use of OCHAs may be broadly grouped into 3 categories: direct mass effects [5], granuloma formation sometimes mimicking tumours [6] and pseudoabscess [7]. An earlier prospective study of Gelita-Cel OCHAs administered at mediastinoscopy suggested that the material was absorbed by day 14 [8]. Foamy macrophage or foam cell deposition was not seen in this study, which examined node stations at lung resection where OCHA materials were left at a prior mediastinoscopy. Foreign body reactions to oxidised regenerated cellulose $\left(\right.$ Surgicel ${ }^{\circledR}$ ), in which the Surgicel mass was invaded by multinucleated 


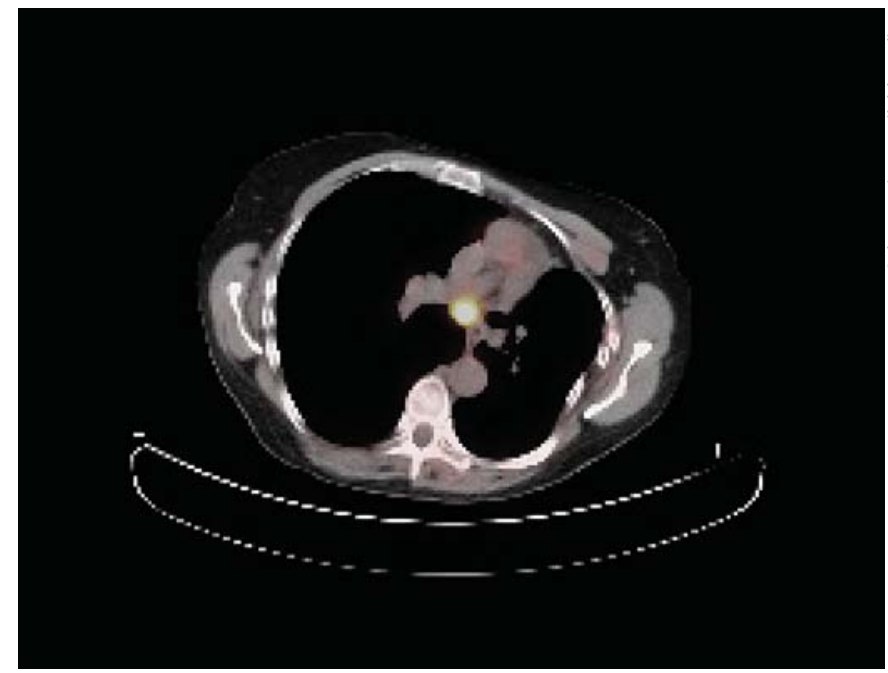

Fig. 2. PET image 5 months after mediastinoscopy and left upper lobectomy, showing new PET-avid subcarinal lymphadenopathy.

giant cells about a week after its application, have been reported [9]. In these reports, a histiocytic reaction with intracytoplasmic materials was seen, without the findings of fat-laden droplets. It is uncertain how macrophages had engulfed oxidized low-density lipoprotein to become foam cells in our case. OCHAs do not contain low-density lipoprotein. Certain pulmonary conditions could induce foamy macrophage collections [10]. It is speculated that these foamy macrophages might have migrated from nearby tissues.

To the best of our knowledge, foamy macrophage or foam cell deposition has not been reported after OCHA use, but the foamy nature of foamy macrophages reflects the substances that they have engulfed/phagocytosed, in this case the cellulose. The macrophages were evaluated both morphologically and by immunohistochemistry, demonstrated by positive expression of CD68 (macrophage marker).

Our findings were reported to the product's manufacturers, who were similarly unaware of previous reports. Foamy macrophage deposition and PET avidity were not seen at the initial mediastinoscopy or staging PET-CT, so both changes had a close temporal relationship to the use of OCHAs. We used an oxidised cellulose product derived from cotton (Gelita-Cel), rather than the more widely used regenerated cellulose products. We do not know if this was significant in the changes seen.

This case suggests a possibility that foamy macrophage formation can occur after the use of OCHAs. We

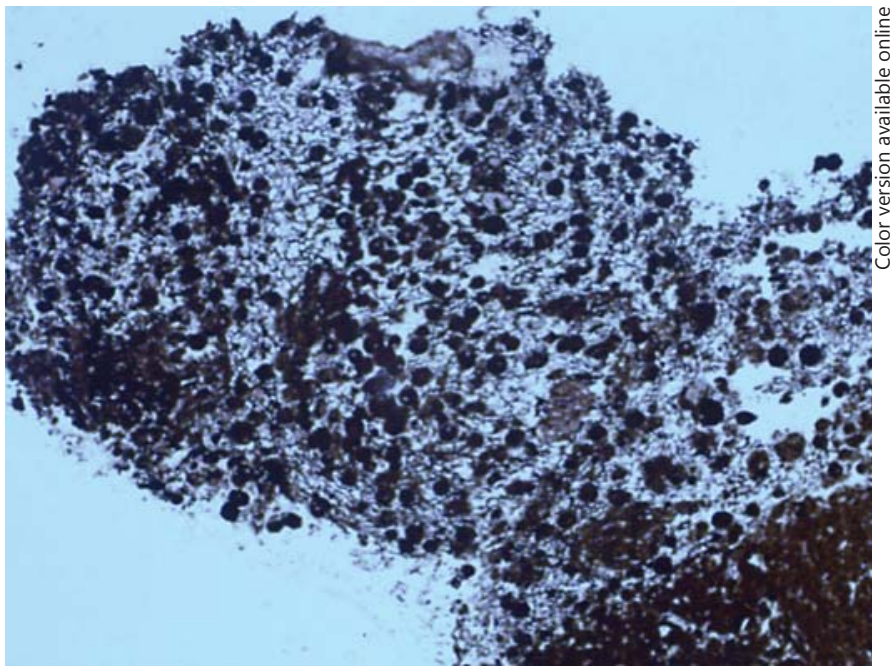

Fig. 3. Microscopic view of foamy macrophage accumulation.

acknowledge that further studies are required to confirm whether this possibility is indeed significant but believe that the association is novel and worthy of reporting so that other clinicians can consider it also. This change mimicked mediastinal node recurrence on both CT and PET-CT imaging. Significant $18-F D G$ avidity is therefore possible in this benign inflammatory process. It is acknowledged that there can be other benign causes of PET avidity after surgery including burnt necrotic tissue after cauterization (not used in this case). The prior use of OCHAs at mediastinoscopy is possibly relevant when investigating a possible mediastinal recurrence of lung cancer. The case also illustrates the well-known utility of noninvasive EBUS-TBNA for investigating mediastinal abnormalities on PET-CT after lung cancer resection. The findings in this case reemphasise the importance of pathological confirmation before considering treatment of suspected malignancy to avoid more invasive unnecessary investigation or expensive invasive treatments with potential side effects and minimise patient anxiety.

\section{Financial Disclosure and Conflicts of Interest}

All authors have no conflict of interest. 


\section{References}

$>1$ Naruke T, Suemasu K, Ishikawa S: Lymph node mapping and curability at various levels of metastasis in resected lung cancer. J Thorac Cardiovasc Surg 1978;76:832-839.

$>2$ Jeyabalan A, Medford AR: Endobronchial ultrasound-guided transbronchial needle aspiration: patient satisfaction under light conscious sedation. Respiration 2014;88:244250.

3 Medford AR: Needle gauge and grey zone analysis in endobronchial ultrasound-transbronchial needle aspiration: the need for more randomised evidence. Respiration 2015;89:438.
4 www.gelitamedical.com/content/gelita-cel ${ }^{\circledR}$. Accessed October 2, 2013.

-5 Teis A, Camara ML, Ferrer E, Romero-Ferrer B: Critical stenosis of pulmonary homograft induced by Surgicel in Ross procedure. Asian Cardiovasc Thorac Ann 2010;18:382-383.

6 Kothbauer KF, Jallo GI, Siffert J, Jimenez E, Allen JC, Epstein FJ: Foreign body reaction to hemostatic materials mimicking recurrent brain tumor. Report of three cases. J Neurosurg 2001;95:503-506.

7 Royds J, Kieran S, Timon C: Oxidized cellulose (Surgicel) based reaction post thyroidectomy mimicking an abscess: a case report. Int J Surg Case Rep 2012;3:338-339.
8 Witte B, Kroeber SM, Hillebrand H, Wolf M, Huertgen M: Cotton-derived oxidized cellulose in minimally invasive thoracic surgery: clinicopathological study. Innovations (Phila) 2013;8:296-301.

9 Kershisnik MM, Ro JY, Cannon GH, Ordonez NG, Ayala AG, Silva EG: Histiocytic reaction in pelvic peritoneum associated with oxidized regenerated cellulose. Am J Clin Pathol 1994;103:27-31.

-10 Yuasa K, Kanazawa T: Foamy alveolar macrophages in various lung disorders, and their origin in rabbit lungs. Nihon Kyobu Shikkan Gakkai Zasshi 1995;33:715-722. 